\title{
OPERACIONALIZAÇÃO E LOGÍSTICA DOS PROCESSOS ADMINISTRATIVOS NA GESTÃO PÚBLICA PARA O ENFRENTAMENTO DA PANDEMIA DE COVID-19
}

OPERATIONALIZATION AND LOGISTICS OF ADMINISTRATIVE PROCESSES IN PUBLIC MANAGEMENT TO FACE THE COVID-19

PANDEMICS

OPERACIONALIZACIÓN Y LOGÍSTICA DE PROCESOS ADMINISTRATIVOS EN LA GESTIÓN PÚBLICA PARA ENFRENTAR LA PANDEMIA DE

COVID-19

Ismael de Vasconcelos Ferreira

Camila Cristina Ripardo Silva 2

Sandra Maria Lopes Vasconcelos 3

Raquel Miranda de Vasconcelos 4

Valdenice Rodrigues Mourão ${ }^{5}$

Giovanni Andrade Menescal 6

Palavras-chave:

Pandemias; Infecções por Coronavirus; Políticas; Planejamento e Administração em Saúde; Administração em Saúde Pública.

Keywords:

Pandemics; Coronavirus Infections; Health Policy, Planning, and Management; Public Health Management.

Palabras clave: Pandemias; Infecciones por coronavirus; Política; Planificación y Gestión de la Salud; Gestión de la Salud Pública.

Submetido: $25 / 11 / 20$

Aprovado: $14 / 12 / 20$

Autor(a) para Correspondência:

Ismael de Vasconcelos Ferreira Rua Francisco Bezerra de Vasconcelos, 507 - Junco, Sobral - CE CEP: 62030-220 Email:ismaelferreira@sobral.ce.gov.br

\section{RESUMO}

0 presente artigo objetiva apresentar o relato da experiência vivenciada pela Coordenadoria Administrativo-Financeira da Secretaria da Saúde de Sobral, sob o olhar das células de gerência, no que concerne ao enfrentamento da pandemia do novo coronavírus no município. Tem como fonte de dados os registros disponiveis nos sistemas de informação e controle das gerências Financeira, Gestão de Pessoas, Logística e Patrimônio, Infraestrutura e Manutenção de Equipamentos e Transportes, compreendendo o período de março a novembro de 2020. A experiência aqui sistematizada tem o potencial de se configurar como um guia para o auxílio de gestores públicos no que concerne aos processos administrativos necessários em meio a novas situações que requeiram tais ações.

1. Graduado em Teologia com Doutorado em Ciência da Religião pela Universidade Federal de Juiz de Fora (UFJF). Coordenador Administrativo-Financeiro da Secretaria da Saúde de Sobral(2018-2020). E-mail: ismaelferreira@ sobral.ce.gov.br. Orcid: https://orcid.org/0000-0002-3958-9946.

2. Graduada em Administração com MBA em Finanças, Controladoria e Auditoria pela Universidade Estadual Vale do Acaraú (UVA). Gerente da Célula Financeira da Secretaria da Saúde de Sobral. E-mail: camilaripardo@sobral.ce.gov. br. Orcid: https://orcid.org/0000-0001-8513-6641.

3. Graduada em Gestão e Serviços de Saúde pela Universidade Estadual Vale do Acaraú (UVA). Gerente da Célula de Gestão de Pessoas da Secretaria da Saúde de Sobral. E-mail: sandravasconcelos@sobral.ce.gov.br. Orcid: https:// orcid.org/0000-0002-3460-4684.

4. Graduada em Ciências Contábeis com Especialização em Administração Pública pela Universidade Estadual Vale do Acaraú (UVA). Gerente da Célula de Logística e Patrimônio da Secretaria da Saúde de Sobral. E-mail: raquelmiranda@sobral.ce.gov.br.0rcid: https://orcid.org/0000-0001-9316-2591.

5. Graduada em Tecnologia da Construção Civil (Edificações) com Especialização em Gestão em Políticas Públicas pela Universidade Estadual Vale do Acaraú (UVA). Gerente da Célula de Infraestrutura e Manutenção de Equipamentos da Secretaria da Saúde de Sobral. E-mail: valdenice.mourao@sobral.ce.gov.br. Orcid: https://orcid. org/0000-0002-3077-140X.

6. Gerente da Célula de Transportes da Secretaria da Saúde de Sobral. E-mail: celiomenescal@sobral.ce.gov.br. Orcid: https://orcid.org/0000-0002-2053-6879

Certificação de redação científica: Central das Revisões. Edição de texto: Karina Matos. Preparação de original: Livia Lingerfelt 


\section{ABSTRACT}

This article aims to present the report of the experience lived by the Coordenadoria Administrativo-Financeira of the Secretaria da Saúde de Sobral (Administrative-Financial Coordination of the Department of Health of Sobral), under the supervision of management units, in regard to the new coronavirus pandemics in the municipality. Its data source are the records available in the information and control systems from the Financial Department; Management of People, Logistics, and Assets; Infrastructure and Maintenance of Equipment and Transportation, covering the period from March to November 2020. The experience systematized here has the potential to be configured as a guide to help public managers with regard to the necessary administrative processes in the midst of new situations that require such actions.

\section{RESUMEN}

Este artículo tiene como objetivo presentar el relato de la experiencia vivida por la Coordinación AdministrativaFinanciera de la Secretaría de Salud de Sobral, bajo la mirada de las células de gestión, en cuanto al enfrentamiento de la pandemia del nuevo coronavirus en el municipio. Su fuente de datos son los registros disponibles en los sistemas de información y control de las áreas Financiera, Gestión de Personas, Logística y Patrimonial, Infraestructura y Mantenimiento de Equipos y Transporte, abarcando el período de marzo a noviembre de 2020. La experiencia aquí sistematizada tiene la potencial para configurarse como una guía para ayudar a los administradores públicos con respecto a los procesos administrativos necesarios en medio de nuevas situaciones que requieran tales acciones.

\section{INTRODUÇÃO}

A pandemia do novo coronavírus (Covid-19) surpreendeu o mundo. Apesar de ter sinalizado o seu início em dezembro de 2019, em Wuhan, na província de Hubei, na China, países e organizações não estavam preparados para seus impactos, que se mostraram tão expressivos e fatais. No Brasil, o primeiro caso foi confirmado em fevereiro de $2020^{1}$. No Ceará, a partir do primeiro caso suspeito (mas não confirmado), notificado ainda em janeiro de 2020, em Sobral ${ }^{2}$, deu-se início a procedimentos de orientação e discussão quanto a planos de contingência para o possivel enfrentamento da doença. A partir daí, compreendeu-se que a chegada efetiva no município de Sobral, caso analisado neste texto, era iminente.

0 Sistema de Saúde de Sobral é bastante complexo. Sede de macrorregião, composta por 55 municípios, que somam mais de um milhão de habitantes, detém em seu próprio sistema municipal especificidades que o tornam sui generis na atenção à saúde: $100 \%$ de abrangência na Atenção Básica, com 37 Unidades Básicas de Saúde (UBS), rede de saúde mental composta por dois Centros de Atenção Psicossocial (Caps AD e Geral) e uma Unidade de Acolhimento Adulto, Centro de Especialidades Médicas e Odontológicas, Centro de Referência em Infectologia, Centro de Reabilitação Física, Unidade de Pronto-Atendimento 24 horas (UPA) e Serviço de Atendimento Móvel de Urgência (SAMU). Sua abrangência ainda compreende zonas distritais e rurais do município, prestando assistência por meio de UBS em 14 localidades situadas a 45 quilômetros (média de distância dos 14 distritos a Sobral) ${ }^{3}$.

A manutenção operacional e logística desse sistema por si só demanda atenção e planejamento constantes, mas com o avanço da pandemia no município, que teve seu efetivo enfrentamento iniciado em 16 de março de 2020, com o estabelecimento do estado de emergência, através do decreto municipal $n .^{0} 2.371^{4}$, após o aumento do número de casos suspeitos no Estado e a confirmação dos primeiros casos no Ceará, em março de $2020^{5}$, passou-se a revisar os planejamentos realizados e a tomar decisões mais rápidas, sobretudo quando o município já havia assumido a gestão de um hospital geral (Hospital Dr. Estevam Ponte) e decidiu abrir um hospital de campanha numa clínica particular que se encontrava desativada no município (Clínica Dr. Alves).

A reorganização da rede operacional e logística, bem como sua implementação neste novo cenário pandêmico, é o que será apresentado a seguir neste texto, sob a condição de relato de experiência. Ele é fruto das experiências vivenciadas no âmbito da Coordenadoria Administrativo-Financeira da Secretaria Municipal da Saúde (SMS) e suas gerências Financeira, Gestão de Pessoas, Logística e Patrimônio, Infraestrutura e Manutenção de 
Equipamentos e Transportes, além do apoio do Instituto para Gestão em Saúde, organização social que presta serviços à Secretaria da Saúde e que atuou de modo significativo na efetivação das ações de enfrentamento da pandemia. Há também o apoio interinstitucional da Secretaria de Ouvidoria, Gestão e Transparência, Secretaria da Infraestrutura, Secretaria do Orçamento e Finanças e Secretaria da Educação, que serão contempladas direta ou indiretamente durante os relatos que seguirão.

objetiva-se, portanto, com este relato de experiências, o registro das ações realizadas para o enfrentamento da pandemia do SARS-CoV-2 (sigla derivada do inglês: severe acute respiratory syndrome coronavirus 2), a fim de garantir uma memória, mas, também, um guia minimamente adequado para a consulta sobre como atuar, numa gestão pública, em cenários semelhantes ao vivido atualmente. As experiências estarão encadeadas de acordo com as gerências responsáveis por cada área, em ordem de abrangência, mas não isoladamente, haja vista a integração entre as áreas ser constante e necessária, sobretudo no momento registrado.

\section{METODOLOGIA}

As experiências aqui relatadas resultaram das ações inerentes às gerências da Coordenadoria Administrativo-Financeira: Financeira, Gestão de Pessoas, Logística e Patrimônio, Infraestrutura e Manutenção de Equipamentos e Transportes. Essas gerências estão dispostas na estrutura da Secretaria da Saúde, ligadas à coordenadoria mencionada (órgãos de execução instrumental) e atuam na resolução de processos meio, dando suporte direto às unidades de assistência à saúde, por meio de suas especificidades, conforme descrito no regulamento da Secretaria Municipal da Saúde ${ }^{6}$. 0 registro e a discussão desses relatos de experiências ocorreram a partir das informações disponíveis nas gerências citadas,

\section{"A geração ou aprimoramento de qualquer bem ou serviço público é um ganho social significativo diante de tudo o que possa representar para a população"}

catalogadas em sistemas de informação, planilhas e anotações gerais, considerando o período de março a novembro de 2020. A disposição das experiências será apresentada a seguir, separadamente por cada gerência, tendo sido estimulada para considerar ações e quantitativos, estes quando existirem. No mais, as discussões registram as percepções das gerências a fim de detalhar as ações realizadas, que aqui foram chamadas de "operacionalização".

\section{RESULTADOS E DISCUSSÃO}

\section{Operacionalização financeira e orçamentária}

A geração ou aprimoramento de qualquer bem ou serviço público é um ganho social significativo diante de tudo o que possa representar para a população. Entretanto, é imperativo destacar que qualquer aquisição feita pela Administração Pública precisa estar alinhada aos instrumentos de planejamento que compõem o Orçamento Público ${ }^{7}$. Nesse sentido, a execução orçamentária e financeira do exercício de 2020, da Secretaria Municipal da Saúde de Sobral (SMSS), caracterizou-se pelo desafio de adequar o orçamento municipal ao recebimento de receitas e o adequado registro de despesas vinculadas aos esforços realizados no combate à pandemia do SARSCov-2.

Os aspectos teóricos e práticos para a operacionalização das adequações que se fizeram necessárias junto ao orçamento da saúde tiveram acentuada relevância, pois propiciaram as condições adequadas para o cumprimento das decisões tomadas pelo Escritório de Crise logo que a pandemia começou a despontar no município, garantindo coesão e observância às normas que conduzem os gastos públicos.

A imprevisibilidade inicial sobre a garantia de verbas específicas de suporte financeiro por parte dos órgãos federais e estaduais para o enfrentamento da Covid-19, apesar de causar instabilidade, não foi objeto de arrefecimento diante das aquisições de bens e serviços que se fizeram necessárias. Obviamente, desde as primeiras informações acerca da lida com a pandemia, as aquisições de insumos começaram a acontecer, mesmo que de maneira mais tímida, para que se pudesse mensurar como as compras iriam se comportar, tanto em volume quanto em qualidade.

Anteriormente a qualquer medida de organização orçamentária, as despesas foram registradas nas rubricas orçamentárias disponíveis no orçamento 
ordinário da Secretaria Municipal da Saúde. Antes do recebimento dos primeiros recursos para o enfrentamento da Covid-19, registrados no Fundo Nacional de Saúde, no grupo "Coronavírus (Covid-19)"8, todo o custeio das ações foi mantido pelos recursos ordinários da União, por meio do Sistema Único de Saúde (SUS), disponibilizados mensalmente pelo Fundo Nacional de Saúde (FNS), através do até então bloco de Custeio das Ações e dos Serviços Públicos de Saúde, que se subdividem nos componentes da Atenção Básica, Atenção de Média e Alta Complexidade, Vigilância em Saúde e Assistência Farmacêutica, além de recursos do tesouro municipal, transferidos e aplicados em saúde por meio da arrecadação de impostos e transferências constitucionais e legais.

Para o entendimento holístico das etapas que foram atravessadas pela excepcionalidade do momento pandêmico, cabe ressaltar que o processo de planejamento do orçamento público é composto por três principais etapas previstas na legislação: Plano Plurianual, Lei das Diretrizes Orçamentárias (LDO) e Lei Orçamentária Anual ( $L O A)^{9}$. A inviabilidade de se seguir a cronologia padrão de planejamento não tornou a execução orçamentária inoperante, tendo em vista que todos os gastos oriundos das atividades de combate à pandemia foram minimamente estimados e estudados, dada a urgência na aplicação das medidas de enfrentamento.

0 passo seguinte foi preconizar tais despesas no Plano de Contingência criado junto ao Escritório de Crise, instituído no âmbito da Secretaria da Saúde, que demarcou as iniciativas em prol das soluções cabíveis para o momento. A partir de então, tais despesas passaram a integrar também a Programação Anual de Saúde 2020 (PAS), ferramenta de planejamento que se projeta na Lei Orçamentária Anual, conforme orientação da Confederação Nacional dos Municípios (CNM), por meio da nota técnica $n .{ }^{\circ} 23-A$ de 13 de abril de $2020^{10}$. Para além da instrumentalização do orçamento, a nota técnica também frisava que os recursos transferidos estariam livres para a utilização finalística com a Covid-19, desde que registrados corretamente por meio da criação de programas de trabalho ou ação orçamentária, bem como fonte de despesas específicas ${ }^{11}$.

Logo após as definições da etapa anterior, iniciouse a elaboração das modificações no orçamento vigente, ancorada nos esclarecimentos da nota do Conselho Nacional de Secretarias Municipais de Saúde (CONASEMS), de 3 de abril de 2020, que demonstrava

\section{"A inviabilidade de se seguir a cronologia padrão de planejamento não tornou a execução orçamentária inoperante"}

o detalhamento de todo o amparo legal para as alterações a serem feitas no orçamento vigente ${ }^{12}$, instruções estas endossadas pela nota técnica $n .^{\circ}$ 24, de 17 de abril de 2020, da $\mathrm{CNM}^{13}$, que, dentro de um contexto mais amplo, abordou todo o fluxo de financiamento do SUS e a proposta de transposição e transferência de saldos financeiros trazidos pela Lei Complementar 172/202014. Sendo assim, foram tomadas medidas necessárias para encaminhamento ao legislativo das alterações e inclusões feitas na LOA de $2020^{15}$, em que a funcional programática definida pela SMS, em parceria com a Secretaria do Orçamento e Finanças, adotou a subfunção 122 - Administração Geral, programa 073 - "Atenção à Saúde: Eficiente e Efetiva" e projeto atividade 1360 - "Ações e Serviços de Enfrentamento ao Covid-19", constantes em decreto municipal ${ }^{16}$.

Ainda no mês de março, o município de Sobral recebeu o primeiro recurso destinado diretamente para o combate à Covid-19, por meio da Portaria n..$^{4} 480^{17}$, de 23 de março de 2020, que destinou R\$ 593.375,40 para o município, sinalizando a iniciativa do Ministério da Saúde (MS) em dar suporte aos Estados e Municípios na forma de incentivos financeiros destinados a objetivo em questão. A referida verba direcionava-se para o custeio das ações de enfrentamento, tendo como referencial uma distribuição per capta para cada município. Para além dessa primeira iniciativa, outras portarias de habilitação e adesão de serviços foram publicadas por parte do MS, possibilitando que os municípios se organizassem para o recebimento de verbas futuras, caso lograssem êxito nas habilitações.

Nos meses subsequentes, o volume de recursos transferidos pela União foi ganhando mais robustez. Logo no mês de abril, somouse ao bloco de custeio o importante ingresso de $\mathrm{R} \$ 10.639 .462,93$, vinculados à portaria $n .^{\circ}$ $774 / 2020^{18}$, para despesas inerentes ao custeio de ações para o enfrentamento da pandemia e 
que viabilizaram a implantação e funcionamento do Hospital de Campanha Dr. Alves, montado no município. Essa evolução positiva nos repasses configurou maior autonomia e liberdade à Secretaria da Saúde na gestão do cenário pandêmico no período mais crítico da curva de contágios.

Outras importantes portarias trouxeram incentivos financeiros para os hospitais filantrópicos que passaram a atuar de maneira complementar no enfrentamento da emergência. Foram as Portarias n. ${ }^{\circ} 827 / 2020^{19}$, n. $^{0} 1393 / 2020^{20}$, n. $^{\circ} 1448 / 2020^{21} \mathrm{e}$ n. ${ }^{\circ} 1771 / 2020^{22}$ e o repasse estadual por meio da resolução ad-referendum CESAU n. ${ }^{\circ}$ 02/202023, beneficiando a Santa Casa de Misericórdia de Sobral e o Hospital do Coração. Para além dos valores já mencionados, outros importantes repasses federais foram feitos ao município de Sobral através de Emendas Parlamentares e das Portarias $n .^{0}$ $430 / 2020^{24}$, n. $^{0} 1857 / 2020^{25}$, n. $^{\circ} 1666 / 2020^{26}, n^{\circ}$ $2288 / 2020^{27}, n^{\circ} 2405 / 2020^{28}, n^{0} 2222 / 2020^{29}, n .{ }^{\circ}$ 2358/202030, n. ${ }^{\circ} 2516 / 2020^{31}$ e n. ${ }^{\circ} 2624 / 2020^{32}$, em que algumas possuem destinação específica dentro do cenário da pandemia e outras têm prazo de execução limitado ao exercício vigente. Até o mês de outubro de 2020, essas portarias somam a importância de $R \$$ $52.321 .234,82$. Este valor encontra-se devidamente categorizado e registrado, já tendo sido em sua maior parte aplicado com lisura e transparência na plena execução dos recursos destinados ao combate à Covid-19.

Cabe ressaltar também os recursos transferidos pelo tesouro municipal, advindos da Lei Complementar n. ${ }^{0} 173 / 2020^{33}$, que estabeleceu auxílio financeiro ao município para ações de enfrentamento da pandemia. Diferentemente dos valores transferidos pelo SUS, estes puderam ser utilizados para a aquisição de despesas de capital, ou seja, viabilizaram o investimento em equipamentos imprescindíveis frente ao combate à Covid-19.

A atualização mais recente no orçamento partiu de uma exigência da STN, por meio da Portaria $n .^{\circ}$ $394^{34}$, de 17 de julho de 2020, que resolveu criar fonte de recurso específica para a natureza de despesa federal relacionada a gastos com a Covid-19. Em atendimento ao disposto, foi feita nova alteração na LOA de $2020^{35}$ para a inclusão da nova fonte, assim como o remanejamento de saldo orçamentário na fonte anterior dentro de cada natureza de despesas contínuas no projeto criado para concentrar tais gastos.

A execução orçamentária e financeira dos

\section{"Evolução positiva nos repasses configurou maior autonomia e liberdade à Secretaria da Saúde na gestão do cenário pandêmico"}

recursos tratados acima é grandemente desafiadora, não somente pelo volume de organização e de valores financeiros, mas também pela observância precisa nos gastos dentro de um sistema de saúde complexo e que precisou se aprimorar para ampliar a assistência de forma adequada à população.

\section{Operacionalização de gestão de pessoas}

A manutenção do funcionamento regular dos serviços de saúde que não tiveram sua programação significativamente alterada pela pandemia, como as Unidades Básicas de Saúde, tornou-se um imperativo para a célula de Gestão de Pessoas. Devido à pandemia e visando à segurança de profissionais que apresentavam comorbidades, 288 funcionários foram afastados temporariamente dos seus serviços. Além disso, devido à suspensão de muitos serviços de assistência durante esse contexto, seus colaboradores foram transferidos para outros serviços que tiveram de ser fortalecidos. Visando também à redução do contato e convívio de pessoas nas instituições, foi instituído o regime de teletrabalho e escala para alguns serviços da Secretaria da Saúde. Essas ações foram precedidas de normatizações e orientações advindas do Executivo Municipal e da Secretaria de Ouvidoria, Gestão e Transparência, no âmbito municipal, e de normatizações e orientações do Ministério da Saúde, no âmbito federal.

Os profissionais afastados por comorbidades foram assistidos por intermédio de perícias médicas realizadas na Secretaria da Saúde, de modo que foram constatadas as suas respectivas comorbidades; assim, seguindo as normativas da gestão municipal ${ }^{36}$, tiveram garantidos os seus afastamentos por período determinado, sendo necessário o retorno para a reavaliação periódica quando convocados. Com a saída desses profissionais foi necessário realocar outros servidores que não estavam sob o risco de comorbidades, advindos de setores que tiveram seus serviços reduzidos ou suspensos. Ainda assim, muitos 
serviços foram afetados e não puderam ser supridos com a quantidade e especificidade de funções adequadas dos remanejamentos realizados. Com isso, foi solicitado junto à Secretaria da Educação, que está com suas escolas com funcionamento suspenso, - apoio para serviços gerais e controladores de acesso. Atualmente existem 76 funcionários dessa Secretaria prestando apoio nas unidades da Secretaria da Saúde.

Após a reorganização dos serviços de saúde necessários ao enfrentamento da pandemia, a Secretaria suspendeu algumas atividades de assistência e os profissionais envolvidos foram transferidos para outros setores ou atividades, dentro de suas unidades de saúde ou de outras unidades, como os profissionais do Centro de Especialidades Odontológicas, Centro de Reabilitação Física, Núcleo Ampliado de Saúde da Família e Escola de Saúde Pública Visconde de Saboia. Eles passaram a atuar, dentre outras ações, no monitoramento de casos suspeitos e confirmados de Covid-19. Esse remanejamento ocorreu com 40 profissionais. Atualmente, com 0 retorno das atividades dessas unidades que tiveram atendimento suspenso e com a redução de casos suspeitos e confirmados de Covid-19, as ações de monitoramento foram absorvidas pelos profissionais dos próprios Centros de Saúde da Família.

Os serviços que permitiam atividades a distância foram normatizados por intermédio do regime de teletrabalho. Para tanto, a Secretaria da Ouvidoria, Gestão e Transparência publicou portaria ${ }^{37}$ orientando a realização e o monitoramento desses serviços por meio de planos de trabalho validados pelos coordenadores responsáveis e inseridos na plataforma de controle de frequência eletrônica. Foram 38 funcionários que atuaram nessa modalidade durante três meses, que corresponderam ao período de maior intensidade da pandemia no município. Ademais, funcionários com comorbidades e que não foram contaminados permaneceram afastados após consultas realizadas com o médico do trabalho e testes realizados pela Secretaria da Saúde. Aos funcionários que tinham direito a férias e licenças-prêmio foi garantido o gozo, desde que estivessem compreendidos no grupo de pessoas com comorbidades e não tivessem sido contaminados, após exame realizado pelo médico do trabalho.

Para atender às demandas de pessoal surgidas com os afastamentos de profissionais e a abertura de novas frentes de atuação para o enfrentamento da pandemia, foram realizadas contratações de 105

\section{"Os serviços que permitiam atividades a distância foram normatizados por intermédio do regime de teletrabalho"}

profissionais que atuaram, em sua maioria, nos hospitais que agora integram a Rede de Assistência à Saúde (RAS) do município.

Inicialmente, para o hospital intervencionado pelo município ainda pouco antes do estabelecimento do período de emergência na cidade, o Hospital Dr. Estevam Ponte ${ }^{38}$, foram admitidos 105 profissionais através de contratação direta, inicialmente por força do decreto de intervenção e, posteriormente, por meio de seleção pública para o período em que o hospital estiver intervencionado (até 31 de janeiro de 2021). Atualmente (novembro de 2020) existem 107 profissionais contratados no referido hospital intervencionado, que também passou a ser referência em leitos retaguarda de enfermaria para pacientes com suspeita ou confirmação de Covid-19 e realização de exames para a detecção da doença.

A outra parte de profissionais contratados para atuarem no enfrentamento da pandemia (73 profissionais) foi programada por meio da criação de funções específicas para profissionais que atuaram no Hospital de Campanha Dr. Alves ${ }^{39,40}$. Para tanto, foi realizada seleção pública conjunta para este hospital e o Hospital Dr. Estevam Ponte, garantindo também um cadastro reserva desses profissionais. Dada a excepcionalidade do período, que demonstrou a indisponibilidade de profissionais habilitados para determinadas atividades, foram realizadas novas seleções para cargos específicos que não haviam sido preenchidos. Em paralelo, o Instituto para Gestão em Saúde também realizou seleção e contratação de profissionais para atuarem temporariamente nas unidades de saúde da Secretaria, bem como no Hospital de Campanha Dr. Alves, a fim de suprir demandas emergenciais e circunstanciais de profissionais, considerando que o referido hospital dispõe de dez leitos de UTI para atendimento exclusivo a pacientes com Covid-19.

Todo esse processo foi seguido dos procedimentos administrativos necessários para a garantia dos princípios da administração pública. Daí que o 
comparecimento presencial dos profissionais para a entrega de documentação; a realização do exame para emissão do Atestado de Saúde 0cupacional, realizado pelo médico do trabalho da Secretaria da Saúde; o procedimento para abertura de conta corrente e os trâmites para a publicação dos contratos no Diário oficial do Município foram seguidos rigorosamente, sendo ainda hoje monitorados devido à circunstância temporal dos contratos.

\section{Operacionalização logística e patrimonial}

A pandemia de Covid-19 chegou à Secretaria da Saúde de Sobral juntamente com a necessidade de encampação do Hospital Dr. Estevam Ponte. Conforme já informado anteriormente, essa instituição de saúde foi intervencionada pelo município ${ }^{41}$. Para tanto, a revisão dos contratos de serviços e fornecimento de materiais teve de ser revista com a realização de aditivos aos contratos, bem como novos processos de aquisição, realizados sobretudo por meio de dispensa de licitação. Com isso, o atendimento a esse novo equipamento se tornaria possível sem haver prejuízo ao funcionamento do hospital ante à intervenção.

Simultaneamente, realizaram-se os processos para as ações de enfrentamento da pandemia, que se apresentavam cada vez mais urgentes, dentro de uma situação que não havia precedentes na história da Secretaria da Saúde de Sobral. Juntouse às 45 unidades de saúde que permaneceram funcionando durante a pandemia o Hospital de Campanha Dr. Alves, que demandou ainda mais planejamento por parte da gerência de Logística e Patrimônio. 0s quantitativos de materiais pareciam sempre insuficientes ou suscitavam dúvidas quando comparados a fornecimento regular para as unidades de saúde existentes. Contudo, logo eram confirmados e, por vezes, superavam o estimado inicialmente, carecendo da repetição dos processos de aquisição para suprir o estoque. Observou-se um aumento significativo nos materiais de limpeza, sendo seguido dos de expediente e de gêneros alimentícios. Estes últimos, devido ao serviço de nutrição e dietética existente à época no Hospital Dr. Estevam Ponte. Acerca dos materiais de limpeza, graças à expertise da Secretaria da Saúde em gerir esses itens para limpeza e higienização de ambientes e equipamentos, foi também dado apoio a outras Secretarias quanto à escolha de produtos e suas aquisições; nesse caso, visando ao enfrentamento da pandemia em outros equipamentos da gestão municipal.

\section{"Durante a pandemia, foi mantida regularmente a dispensação de materiais, dentro $e$ fora da programação estabelecida"}

Com o advento do Hospital de Campanha Dr. Alves e a reestruturação do Hospital Dr. Estevam Ponte, o levantamento de equipamentos e material permanente para a sua adequada montagem também demandou da célula de Logística e Patrimônio ações coordenadas e emergenciais. A identificação desses materiais necessários e sua disponibilidade em estoque ou sua viabilidade para a aquisição (existência de contratos vigentes) demandou desta célula, em constante articulação com a célula financeira e de infraestrutura, o planejamento para a aquisição e distribuição, respectivamente, dos materiais devidos às duas unidades hospitalares. Em termos quantitativos, foram dispensados 34 computadores, três impressoras, 306 equipamentos hospitalares, dentre outros materiais de consumo necessários à estruturação dos hospitais, e adquiridos 61 equipamentos hospitalares exclusivamente para os dois hospitais intervencionados para o enfrentamento do novo coronavírus.

Durante a pandemia, foi mantida regularmente a dispensação de materiais, dentro e fora da programação estabelecida para essa dispensação, visando sempre à manutenção do funcionamento das unidades de saúde, não havendo registros de desabastecimento ou falta de itens essenciais ao enfrentamento da pandemia, como materiais de limpeza.

\section{Operacionalização e logística de infraestrutura}

Para garantir a estrutura física necessária ao funcionamento das unidades de saúde existentes no município e, principalmente, as novas unidades hospitalares intervencionadas para o enfrentamento da pandemia de Covid-19, a célula de infraestrutura e manutenção de equipamentos realizou direta e indiretamente ações de intervenção e melhoria em todas as unidades dedicadas a esse enfrentamento. A atuação dessa célula se deu porintermédio da força de trabalho existente, composta de profissionais aptos 
às mais diversas necessidades que se apresentavam, e da contratação e acompanhamento de serviços especializados em manutenção predial, instalação hidráulica, elétrica e eletrônica e de informática.

As intervenções nas unidades de saúde foram iniciadas com a instalação de dispensers de álcool em gel, adequações de infraestrutura interna (manutenção predial) e configuração e orientação para uso de ferramentas de internet (utilização do Whatsapp Web para fins de monitoramento dos casos suspeitos e confirmados de Covid-19 pelas UBS).

Por vezes, simultaneamente, o atendimento à estruturação dos dois hospitais intervencionados ocorreu através de frentes de trabalho que compunham a reforma e manutenção predial (reativação de elevador, revisão e reforma de telhados, substituição de lâmpadas e tomadas, estruturação de rede lógica e instalação e revisão de rede hidráulica e hidrossanitária), transporte e instalação de equipamentos (computadores, equipamentos hospitalares e ar condicionado) e contratação de serviços essenciais, como instalação de gerador de energia e empresa especializada em instalação de equipamentos de UTI para o Hospital de Campanha Dr. Alves.

Deve-se ressaltar que mesmo empregando grande força de trabalho a essas duas novas unidades da estrutura da Secretaria da Saúde, o atendimento e acompanhamento de serviços das demais unidades, notadamente as Unidades Básicas de Saúde, mantiveram-se funcionando a contento. Nesse período foram reformadas sete unidades de saúde, estando uma ainda em fase de conclusão, realizando intervenções que viabilizaram também o melhor atendimento à população para o enfrentamento da pandemia.

\section{Operacionalização e logística de transportes}

Como parte das ações de enfrentamento da pandemia de Covid-19, a Secretaria da Saúde não prescindiu de sua frota sanitária para viabilizar a logística de transportes necessária para a assistência à saúde. Durante o período mais crítico do enfrentamento, vivenciado entre os meses de maio e junho, a Secretaria manteve todos os seus 14 distritos providos de ambulâncias, garantindo a segurança dos seus condutores no transporte de pacientes suspeitos ou confirmados de Covid-19 até a sede do município e pacientes com outras necessidades de assistência. Igualmente, a oferta

\section{"Foram contratadas ambulâncias por intermédio de serviço de locação para fortalecer a estratégia de atendimento ao transporte sanitário"}

de carros de apoio manteve-se constante, atendendo distritos, serviços de inspeção (Vigilância Sanitária), vacinação em domicílio e viagens excepcionais à cidade de Fortaleza, transportando pacientes que necessitavam do serviço de transporte, regulados pelo Serviço de Apoio ao Cidadão Sobralense.

A manutenção dessa logística deu-se graças ao efetivo serviço mecânico contratado pela célula de transportes, que incluía o contato com oficinas mecânicas que, apesar de funcionarem em horário reduzido devido às normatizações de suspensão de atividades comerciais para a redução do contato entre as pessoas, mantiveram seus serviços minimamente regulares. Com isso, o reparo dos veículos, sobretudo ambulâncias, dava-se de maneira satisfatória, sendo possível manter todas as unidades situadas em distritos guarnecidas do transporte sanitário.

Ainda assim, diante de situações limítrofes que demandavam decisões urgentes, como o transporte adequado de pacientes graves ou não, foram contratadas ambulâncias porintermédio de serviço de locação para fortalecer a estratégia de atendimento ao transporte sanitário. Outrossim, veículos de apoio (carros de passeio) também foram contratados por meio de locação a fim de dar o suporte necessário nesse serviço sensível e essencial à saúde pública. Assim, o atendimento aos usuários do sistema de saúde manteve-se efetivo e satisfatório, apesar da situação de emergência em que se encontrava 0 município, sobretudo no período supracitado. Ao mesmo tempo, essa situação de emergência gerou novas formas de atenção e resolução de problemas com o incremento de ferramentas de monitoramento, controle de veículos e atendimento a demandas regulares e extemporâneas.

\section{CONCLUSÃO}

Com a apresentação e o registro das ações realizadas pela Coordenadoria AdministrativoFinanceira, por meio da vivência e experiência de 
suas células de gerência, é possível vislumbrar as dinâmicas que esta coordenação executou, cada uma ao seu modo, com práticas e objetivos específicos distintos, mas voltados para uma ação única: o enfrentamento da pandemia do novo coronavírus. 0 trabalho coordenado e em cooperação trouxe resultados importantes para esse enfrentamento, haja vista a gama de realizações que se acumularam nesse período e que ainda hoje podem ser constatadas. Há que se ressaltar também o apoio de instituições e empresas que demonstraram interesse no auxílio ao enfrentamento da Covid-19 e efetivaram ações de cooperação (doação de materiais e cessão de equipamentos e veículos).

As ações realizadas demonstraram, portanto, a relevância dos processos administrativos dessa coordenadoria, que se mostra necessária à execução de atividades meio, a fim de viabilizar os processos assistenciais desenvolvidos pela Secretaria da Saúde de Sobral. Para tanto, há que se ressaltar a eficiência das gerências ligadas a esta coordenadoria, a qual, apesar dos percalços enfrentados durante a pandemia (que ainda persiste), manteve a contento, dentro das condicionalidades apresentadas, 0 funcionamento dessa Secretaria e contribuiu direta e indiretamente para o enfrentamento da pandemia do novo coronavírus.

\section{CONTRIBUIÇÃO DOS AUTORES}

Ismael de Vasconcelos Ferreira contribuiu com redação, análise e revisão crítica do manuscrito. Camila Cristina Ripardo Silva contribuiu com redação, análise e revisão crítica do manuscrito. Sandra Maria Lopes Vasconcelos contribuiu com redação, análise e revisão crítica do manuscrito. Raquel Miranda de Vasconcelos contribuiu com redação, análise e revisão crítica do manuscrito. Valdenice Rodrigues Mourão contribuiu com redação, análise e revisão crítica do manuscrito. Giovanni Andrade Menescal contribuiu com redação, análise e revisão crítica do manuscrito.

\section{REFERÊNCIAS}

1. Brasil. Brasil confirma primeiro caso da doença [home-page on the internet]. Brasília: Ministério da Saúde; 2020 [cited 2020 Nov 25]. Available from: https://www.gov.br/saude/pt-br/assuntos/noticias/ brasil-confirma-primeiro-caso-de-novo-coronavirus
2. Secretaria da Saúde. Sesa divulga nota técnica para orientar profissionais sobre novo coronavírus [home-page on the internet]. Ceará; 2020 [cited 2020 Nov 25]. Available from: https://www.saude. ce.gov.br/2020/01/29/sesa-divulga-nota-tecnicapara-orientar-profissionais-sobre-novo-coronavirus/

3. Sobral. Plano Municipal de Saúde de Sobral 2018 a 2021. Sobral: Secretaria Municipal da Saúde. Coordenação da Educação na Saúde - Escola de Formação em Saúde da Família Visconde de Saboia; 2017.

4. Sobral. Decreto n. ${ }^{0} 2.371$, de 16 de março de 2020. Diário Oficial do Município [document on the internet], Sobral (2020 Mar 16 [cited 2020 Nov 22]). Available from: http://www.sobral.ce.gov. br/diario/public/files/diario/9bf7ba4ae2931bdf1 4b3a48b5240d141.pdf

5. Secretaria da Saúde. Boletim Epidemiológico. Doença pelo novo coronavírus (COVID-19) [homepage on the internet]. Ceará; 2020 [cited 2020 Nov 25]. Available from: https://www.saude. ce.gov.br/wpcontent/uploads/sites/9/2018/06/ boletim n13 COVID 14 mar 20 v2.pdf

6. Sobral. Decreto $n^{0} 2346$, de 7 de fevereiro de 2020. Diário Oficial do Município [document on the internet], Sobral (2020 Feb 7 [cited 2020 Nov 22]). Available from: http://www.sobral.ce.gov. br/diario/public/files/diario/b3e7a48e267a52df25 1538c399668d13.pdf

7. Controladoria Geral da União. Portal da Transparência - Orçamento Público [document on the internet]. Brasília: CGU; 2020 [cited 2020 Nov 22]. Available from: http://www.portaltransparencia. gov.br/entenda-a-gestao-publica/orcame nto-publico

8. Fundo Nacional de Saúde. Consulta [home-page on the internet]. Brasília: Ministério da Saúde; 2020 [cited 2020 Nov 22]. Available from: https:// consultafns.saude.gov.br/\#/detalhada/acao

9. Brasil. Manual de Contabilidade Aplicada ao Setor Público. 8. ed. Brasília: Secretaria do Tesouro Nacional; 2018.

10. Confederação Nacional de Municípios. Nota técnica n. ${ }^{\circ} 023 / 2020$ [document on the internet]. Brasília: CNM; 2020 [cited 2020 Nov 22]. Available from: https://www.cnm.org.br/cms/biblioteca/ NT n.23.2020 Contabilizacao de transferencias fundo a fundo para acoes emergenciais da saude no combate COVID-19.pdf

11. Confederação Nacional de Municípios. Nota técnica n. ${ }^{0}$ 024/2020 [document on the internet]. Brasília: CNM; 2020 [cited 2020 Nov 21]. Available from: https://www.cnm.org.br/cms/biblioteca/ 
NT n.24.2020-Aplicacao-dos-recursos-SUS-parao-enfrentamento-da-Covid-19-e-transposicao-etransferencia-de-recursos-LC $1722020 \quad 2020.07 .02$. $\underline{p d f}$

12. Conselho Nacional de Secretarias Municipais de Saúde. COVID-19. “E agora, Olga, o que eu faço? Orçamento e planejamento [document on the internet]. Brasília; 2020 Brasília; 2020 [cited 2020 Nov 22]. Available from: https://www.conasems. org.br/wp-content/uploads/2020/04/2020 NotaCONASEMS-.pdf

13. Confederação Nacional de Municípios. Nota técnica n. ${ }^{\circ}$ 024/2020 [document on the internet]. Brasília; 2020 [cited 2020 Nov 21]. Available from: https://www.cnm.org.br/cms/biblioteca/ NT n.24.2020-Aplicacao-dos-recursos-SUS-parao-enfrentamento-da-Covid-19-e-transposicaoe-transferencia-de-recursosLC 1722020 2020.07.02.pdf

14. Conselho Nacional de Secretarias Municipais de Saúde. COVID-19. E agora, Olga, o que eu faço? L C 172/2020 - PLP 232/40 [document on the internet]. Brasília; 2020 [cited 2020 Nov 21]. Available from: https://www.conasems.org.br/wp-content/ uploads/2020/04/NOTASALD0S2020 $04 \quad 16 \quad 0$ lga PLP232 LC172.pdf

15. Sobral Prefeitura Municipal de. "Diário Oficial do Município". Available from: http://www.sobral. ce.gov.br/diario/public/files/diario/989532000c5ef 322ed729ed3e0510da8.pdf

16. ${ }^{5}$ obral. Lei Municipal n. ${ }^{\circ} 1.607$, de 02 de fevereiro de 2017 e regulamentado pelo Decreto Municipal $n .^{\circ}$ 1961, de 22 de novembro de 2017. Diário Oficial do Município [document on the internet], Sobral (2020 Feb 2 [cited 2020 Nov 22]). Available from: http:// www.sobral.ce.gov.br/diario/public/files/diario/9bf 7ba4ae2931bdf14b3a48b5240d141.pdf

17. Brasil. Portaria n. ${ }^{\circ} 480$, de 23 de março de 2020. Diário Oficial da União [document on the internet], Brasília (2020 Mar 23 [cited 2020 Nov 22]). Available from: Available from: https://www. in.gov.br/en/web/dou/-/portaria-n-480-de-23-demarco-de-2020-249621650

18. Brasil. Portaria n..$^{774}$, de 9 de abril de 2020. Diário Oficial da União [document on the internet], Brasília (2020 Feb 7 [cited 2020 Nov 22]). Available from: Available from: https://www.in.gov.br/ en/web/dou/-/portaria-n-774-de-9-de-abrilde-2020-251969714.

19. Brasil. Portaria n. ${ }^{\circ} 827$, de 15 de abril de 2020. Diário Oficial da União [document on the internet], Brasília (2020 Apr 15 [cited 2020 Nov 22]). Available from: Available from: https://www.in.gov. br/en/web/dou/-/portaria-n-827-de-15-de-abrilde-2020-252725909
20. Brasil. Portaria n. ${ }^{0} 1.393$, de 21 de maio de 2020. Diário Oficial da União [document on the internet], Brasília (2020 May 21 [cited 2020 Nov 26]). Available from: Available from: https://www. in.gov.br/en/web/dou/-/portaria-n-1.393-de-21-demaio-de-2020-258046968

21. Brasil. Portaria n. ${ }^{0} 1.448$, de 29 de maio de 2020. Diário Oficial da União [document on the internet], Brasília (2020 May 29 [cited 2020 Nov 22]). Available from: Available from: https://www. in.gov.br/en/web/dou/-/portaria-n-1.448-de-29de-maio-de-2020-*-259490693.

22. Brasil. Portaria n. ${ }^{0} 1.771$, de 15 de julho de 2020. Diário Oficial da União [document on the internet], Brasília (2020 July 15 [cited 2020 Nov 22]). Available from: https://www.in.gov.br/ en/web/dou/-/portaria-n-1.771-de-15-de-julhode-2020-267034884

23. Ceará. AD Referendum/CESAU n. ${ }^{0}$ 02/2020. Diário Oficial do Estado [document on the internet], Brasília (2020 Fev 15 [cited 2020 Nov 22]). Available from: http://imagens.seplag.ce.gov.br/PDF/20201105/ do20201105p02.pdf\#page $=82$

24. Brasil. Portaria n. ${ }^{\circ}$ 430, de 19 de março de 2020. Diário Oficial da União [document on the internet], Brasília (2020 July 15 [cited 2020 Nov 22]). Available from: https://www.in.gov.br/en/web/dou/-/ portaria-n-430-de-19-de-marco-de-2020-249027837

25. Brasil. Portaria n. ${ }^{0} 1.857$, de 28 de julho de 2020. Diário Oficial da União [document on the internet], Brasilia (2020 July 28 [cited 2020 Nov 22]). Available from: https://www.in.gov.br/ en/web/dou/-/portaria-n-1.857-de-28-de-julhode-2020-268993763

26. Brasil. Portaria n. ${ }^{0} 1.666$, de 1 de julho de 2020. Diário Oficial da União [document on the internet], Brasília (2020 July 1 [cited 2020 Nov 20]). Available from: https://www.in.gov.br/en/web/dou/-/ portaria-n-1.666-de-1-de-julho-de-2020-264505695

27. Brasil. Portaria n. ${ }^{0} 2.288$, de 27 de agosto de 2020. Diário Oficial da União [document on the internet], Brasília (2020 Ago 27 [cited 2020 Nov 22]). Available from: https://www.in.gov.br/en/ web/dou/-/portaria-n-2.624/gm/ms-de-28-de-sete mbro-de-2020-280037838

28. Brasil. Portaria n. ${ }^{0} 2.405$, de 16 de setembro de 2020. Diário Oficial da União [document on the internet], Brasília (2020 Sept 16 [cited 2020 Nov 23]). Available from: https://www.in.gov.br/en/ web/dou/-/portaria-n-2.405-de-16-de-setembrode-2020-277907703

29. Brasil. Portaria n. ${ }^{0} 2.222$, de 25 de agosto de 2020. Diário Oficial da União [document on the 
internet], Brasília (2020 Ago 25 [cited 2020 Nov 22]). Available from: https://www.in.gov.br/en/ web/dou/-/portaria-n-2.222/gm/ms-de-25-deagosto-de-2020-274149904

30. Brasil. Portaria n. ${ }^{\circ} 2.358$, de 2 de setembro de 2020. Diário Oficial da União [document on the internet], Brasília (2020 Sept 2 [cited 2020 Nov 23]). Available from: https://www.in.gov.br/en/ web/dou/-/portaria-n-2.358-de-2-de-setembrode-2020-275909887

31. Brasil. Portaria n. ${ }^{0} 2.516$, de 21 de setembro de 2020. Diário Oficial da União [document on the internet], Brasília (2020 Sept 21 [cited 2020 Nov 23]). Available from: https://www.in.gov.br/en/ web/dou/-/portaria-n-2.516-de-21-de-setembrode-2020-278695720

32. Brasil. Portaria n. ${ }^{0}$ 2.624, de 28 de setembro de 2020. Diário Oficial da União [document on the internet], Brasília (2020 Sept 28 [cited 2020 Nov 24]). Available from: https://www.in.gov.br/ en/web/dou/-/portaria-n-2.624/gm/ms-de-28-desetembro-de-2020-280037838

33. Brasil. Lei complementar n. ${ }^{0} 173$, de 27 de maio de 2020. Diário Oficial da União [document on the internet], Brasília (2020 May 27 [cited 2020 Nov 24]). Available from: https://www.in.gov.br/en/ web/dou/-/lei-complementar-n-173-de-27-de-maiode-2020-258915168

34. Brasil. Portaria n. ${ }^{0} 394$, de 29 de maio de 2020. Diário Oficial da União [document on the internet], Brasília (2020 May 29 [cited 2020 Nov 22]). Available from: https://www.in.gov.br/en/web/dou/-/ portaria-n-394-de-29-de-maio-de-2020-259415070

35. Sobral. Lei Municipal n. ${ }^{0} 1.607$, de 02 de fevereiro de 2017 e regulamentado pelo Decreto Municipal n. ${ }^{\circ}$ 1961, de 22 de novembro de 2017. Diário Oficial do Município [document on the internet], Brasília (2017 Fev2 [cited 2020 Nov22]). Available from: http://www. sobral.ce.gov.br/diario/public/files/diario/989532 000c5ef322ed729ed3e0510da8.pdf

36. Sobral. Decreto $n^{0} 2.371$, de 16 de março de 2020. Diário Oficial do Município [document on the internet], Brasília (2020 Mar 16 [cited 2020 Nov 24]). Available from: http://www.sobral.ce.gov. br/diario/public/files/diario/9bf7ba4ae2931bdf14 b3a48b5240d141.pdf

37. Sobral. Portaria n. ${ }^{0} 004 / 2020$ - SEGET, de 17 de março de 2020. Diário Oficial do Município [document on the internet], Brasillia (2020 Mar 17 [cited 2020 Nov 24]). Available from: http:// www.sobral.ce.gov.br/diario/public/files/diario/ 97d8b9ed7bfe9ef533886cc335105fe3.pdf

38. Sobral. Decreto n. ${ }^{0}$ 2.369, de 13 de março de
2020. Diário Oficial do Município [document on the internet], Brasília (2020 Mar 13 [cited 2020 Nov 24]). Available from: http://www.sobral.ce.gov. br/diario/public/files/diario/00ebe56060c94df73 4b04637524ca2eb.pdf

39. Sobral. Decreto n. ${ }^{0}$ 2.387, de 29 de março de 2020. Diário Oficial do Município [document on the internet], Brasília (2020 Mar 29 [cited 2020 Nov 25]). Available from: http://www.sobral.ce.gov. br/diario/public/files/diario/bc88ae180ed744586 de2c7978efa0086.pdf

40. Sobral. Decreto n. ${ }^{\circ} 2.426$, de 9 de maio de 2020. Diário Oficial do Município [document on the internet], Brasília (2020 May 9 [cited 2020 Nov 25]). Available from: http://www.sobral. ce.gov.br/diario/public/files/diario/160dd2614961a 257ada349a038501e52.pdf

41. Sobral. Decreto n. ${ }^{0} 2.369$, de 13 de março de 2020. Diário Oficial do Município [document on the internet], Brasília (2020 Mar 13 [cited 2020 Nov 25]). Available from: http://www.sobral.ce.gov. br/diario/public/files/diario/00ebe56060c94df73 4b04637524ca2eb.pdf
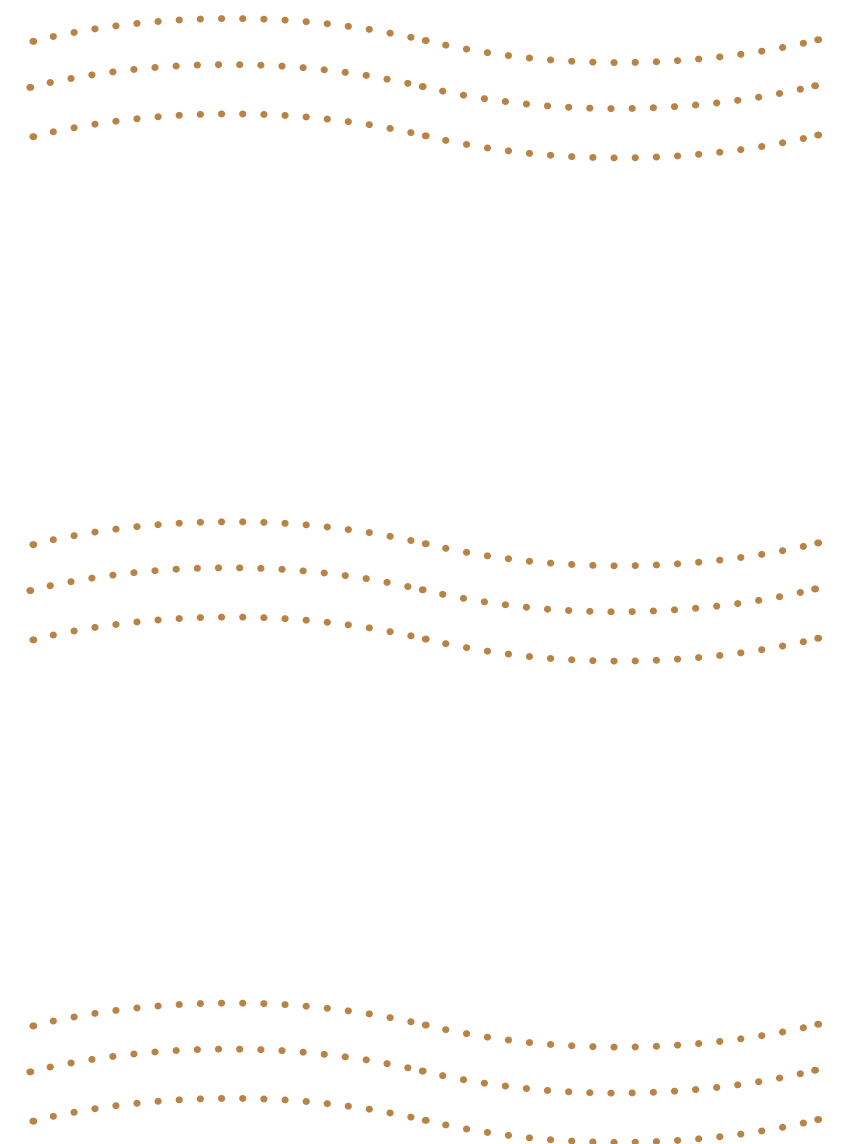